\title{
Finite Element Analysis-Aided Performance Improvement of Circular Coil Assemblies Applied in Electric Vehicle Inductive Chargers
}

\author{
Guodong Zhu, Dawei Gao ${ }^{1}$ \\ ${ }^{1}$ (Corresponding author) State Key Laboratory of Automotive Safety and Energy, School of Vehicle and Mobility, Tsinghua \\ University, Beijing, China.Email: dwgao@mail.tsinghua.edu.cn
}

\begin{abstract}
Summary
Energy efficiency and leakage magnetic field (LMF) are two important issues in inductive chargers. In this work, the maximum achievable coil efficiency and the corresponding LMF strength are formulated as functions of system parameters, and figure of merits (FOM) are proposed for assessing the efficiency and LMF performance of the coil assemblies. The target application is electric vehicle inductive chargers where the LMF is suppressed via passive shielding. The impact of the coil assembly's geometric parameters on both FOMs is examined through a combination of finite element analysis (FEA) simulation and magnetic circuit analysis, and measures to improve the FOMs are studied. Optimization of an exemplary coil assembly within given dimensional limits is conducted and the performance improvement is verified by FEA simulation results.
\end{abstract}

Keywords: efficiency, inductive charger, optimization, simulation, wireless charging

\section{Introduction}

Efficiency improvement [1] and leakage magnetic field (LMF) suppression [2] are two important issues in inductive power transfer (IPT) systems. The conversion between electrical energy and magnetic energy is realized via a pair of coil assemblies, one on the transmitter (TX) side and the other on the receiver (RX) side, hence optimization of the coil assemblies is an important concern in system design.

In a typical electric vehicle inductive charger, which is the target application of this work, a coil assembly contains three parts: an aluminum shielding plate, a ferrite core and a copper winding, hence the coil ESR (equivalent series resistance) is a consequence of all three loss terms. The eddy currents in the aluminum plate result in eddy loss, which is related to the conductivity of aluminum, the incident magnetic flux density and the magnetic field frequency. Ferrite core loss can be estimated using the renowned Steinmetz equation, which is a convenient tool for characterizing magnetic core loss under sinusoidal magnetic field excitations. After the core material is determined, the main influencing factors of core loss are the magnetic flux density and the magnetic field frequency. The power loss in the copper winding is influenced by the conductivity of copper and the unevenness of current density distribution on the wire cross section. Under a given excitation current amplitude, all loss terms are affected by the geometric parameters of the coil assembly. The maximum achievable energy conversion efficiency of the coil assemblies (designated as $\eta_{c}$ and abbreviated as "coil efficiency" in the rest of this paper) is influenced by not only the coil ESRs but also the mutual inductance, and the difficulty in maximizing $\eta_{c}$ lies in the fact that both factors are related to the geometric parameters in complex ways. To objectively assess the efficiency performance of a coil assembly design, figure of merits (FOMs) that reflect the 
combined influence of all relevant factors are a useful criterion [3]. Obviously, the $\eta_{c}$-FOM is heavily reliant on the geometric parameters of the coil assemblies. Measures to improve $\eta_{c}$ via coil assembly optimization have been widely discussed [3-4].

As for LMF suppression, the measures can be divided into two categories: active suppression [2] and passive shielding [5]. In active suppression schemes, extra coils are usually deployed to generate a magnetic field component that partially counteracts the field generated by the TX and RX coils, thus reducing the overall field strength to an acceptable level. Due to the extra hardware and software cost, such methods are more suitable for high-power applications where passive shielding alone is insufficient. By contrast, passive shielding techniques rely on the design of the coil assembly to reduce the leakage magnetic field strength and are widely adopted due to the simplicity and effectiveness. LMF suppression techniques generally have a negative impact on $\eta_{c}$, and there are many researches on striking a balance between $\eta_{c}$ and the LMF strength $[4,6]$.

In this paper, only passive shielding is adopted. Two FOMs are proposed to assess the efficiency performance and the LMF performance, respectively. This work is focused on the ferrite layer structure and the copper winding parameters and aims to reveal the impact of the geometric parameters on the FOMs. Finite element analysis (FEA) together with magnetic circuit analysis are the main tools. Based on FEA simulations and experimental measurements, a manually optimized design is compared with a reference design to demonstrate the improvement in performance.

\section{Performance Evaluation of Coil Assemblies}

\subsection{Efficiency Analysis}

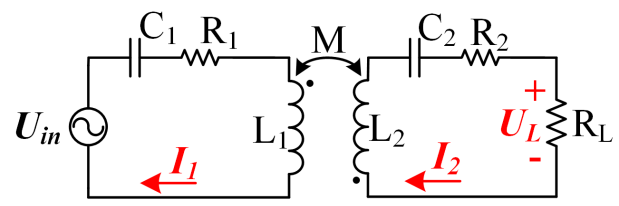

Fig. 1. Simplified schematic of series-series-compensated IPT system.

A simplified schematic of an IPT system with series compensation on both sides is shown in Fig. 1. $\boldsymbol{U}_{\text {in }}$ is the inverter output voltage. $\boldsymbol{U}_{\boldsymbol{L}}$ is the voltage across the load resistance. $\boldsymbol{I}_{\mathbf{1}}$ and $\boldsymbol{I}_{2}$ are the coil currents. $C_{1}$ and $C_{2}$ are the series compensation capacitors. $L_{1}$ and $L_{2}$ are the coil inductances. $\mathrm{M}$ is the mutual inductance. $R_{L}$ is the equivalent AC load resistance. $R_{1}$ and $R_{2}$ are the coil ESRs. Considering that the focus of this work is coil assembly optimization, the power losses in the other components are not considered. Due to the bandpass property of the compensation networks, only the fundamental voltages and currents are utilized in the derivations while the harmonics are neglected. For the sake of simplicity, the coil ESRs are considered in efficiency calculations only.

Methods to maximize $\eta_{c}$ have been analyzed in numerous researches [1]. For the simplified IPT system shown in Fig. 1, the output power $\left(P_{\text {out }}\right)$ is calculated as

$$
P_{\text {out }}=\frac{1}{2} \boldsymbol{I}_{\mathbf{1}} \cdot\left(j \omega M \boldsymbol{I}_{2}\right) \leq \frac{1}{2} \omega M\left|\boldsymbol{I}_{\mathbf{1}}\right| \cdot\left|\boldsymbol{I}_{2}\right| .
$$

The power loss on coil ESRs is calculated as

$$
P_{\text {coil }}=\frac{1}{2}\left(\left|\boldsymbol{I}_{\mathbf{1}}\right|^{2} R_{1}+\left|\boldsymbol{I}_{\mathbf{2}}\right|^{2} R_{2}\right) \geq 2 P_{\text {out }} \frac{\sqrt{R_{1} R_{2}}}{\omega M} .
$$

The conditions for minimizing the $P_{\text {coil }}$-to- $P_{\text {out }}$ ratio are as follows:

$$
\begin{gathered}
\angle \boldsymbol{I}_{1}-\angle \boldsymbol{I}_{2}=90^{\circ}, \\
\left|\boldsymbol{I}_{\mathbf{1}}\right|^{2} R_{1}=\left|\boldsymbol{I}_{\mathbf{2}}\right|^{2} R_{2} .
\end{gathered}
$$

$34^{\text {th }}$ International Electric Vehicle Symposium and Exhibition 
The first condition can be satisfied by maintaining the circuits in full resonance, i.e., $L_{1} C_{1}=L_{2} C_{2}=1 / \omega^{2}$, where $\omega$ is the angular operating frequency of the IPT system. In this work, $\omega=2 \pi \times 85000 \mathrm{rad} / \mathrm{s}$. Equation (2) reveals that the lowest achievable $P_{\text {coil }}$-to- $P_{\text {out }}$ ratio is a function of $\frac{M^{2}}{R_{1} R_{2}}$. In this paper, this ratio is selected as the FOM for assessing the efficiency performance of the coil assemblies and designated as $F O M_{e f f i}$. A larger $F O M_{e f f i}$ corresponds to a higher maximum achievable $\eta_{c}$ value.

\subsection{Leakage Magnetic Field Analysis}

LMF is defined as the magnetic field that users might be exposed to under predefined operating conditions, e.g., the magnetic field in the vicinity of an inductively charged electric vehicle. Under the assumption that the flux lines generated by both coil assemblies are overlapped in the LMF region [2], the LMF strength at a selected observation point under conditions (2-a) and (2-b) is obtained as

$$
B_{L M F}=\sqrt{k_{1}^{2}\left|\boldsymbol{I}_{1}\right|^{2}+k_{2}^{2}\left|\boldsymbol{I}_{2}\right|^{2}}=\sqrt{\frac{2 P_{\text {out }}}{\omega M}} \sqrt{k_{1}^{2} \sqrt{\frac{R_{2}}{R_{1}}}+k_{2}^{2} \sqrt{\frac{R_{1}}{R_{2}}},}
$$

where $k_{1 / 2}$ denotes the magnetic flux density generated by 1 Ampere of current in $L_{1 / 2}$ on the selected observation point. Therefore, the expression $M \cdot\left(k_{1}^{2} \sqrt{\frac{R_{2}}{R_{1}}}+k_{2}^{2} \sqrt{\frac{R_{1}}{R_{2}}}\right)^{-1}$ is selected as the FOM for assessing the LMF performance and designated as $F O M_{L M F}$. A larger $F O M_{L M F}$ corresponds to lower LMF strength at the maximum- $\eta_{c}$ operating point.

Although the highest $B_{L M F}$ occurs in the case of maximum coil misalignment due to the short distance between the ground-side coil assembly and the boundaries of the vehicle, the $F O M_{L M F}$ calculated based on the $k_{1}$ and $k_{2}$ data measured at the zero-misalignment position is also a useful indicator of the LMF performance. Overall, the LMF strength in the maximum-misalignment case has a positive correlation with that in the zeromisalignment case.

\section{Influence of Geometric Parameters}

\subsection{Overall Design and Magnetic Circuit Analysis}

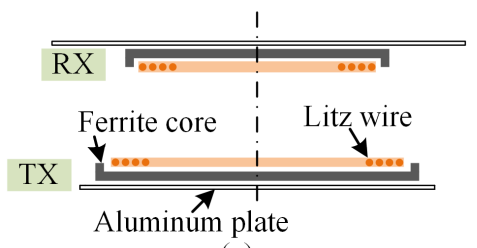

(a)

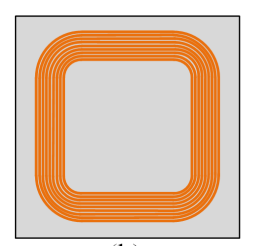

(b)

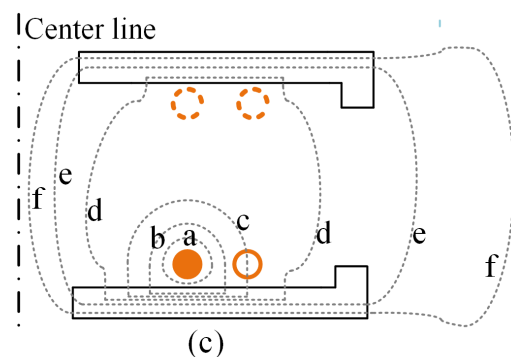

(c)

Fig. 2. Overall design of the coil assemblies and the magnetic flux paths. (a) Front view. (b) Top view. (c) Magnetic flux paths.

As is shown in Fig. 2 (a), a solid ferrite layer is inserted between the planar copper winding and the aluminum shielding plate in the coil assembly. FEA results reveal that under the same ferrite consumption, using an integral ferrite core instead of separate ferrite strips with obvious spaces in between results in the lowest core loss and eddy loss. Protrusions are added along the outer contour of the ferrite layer to improve the mutual inductance and reduce eddy loss in the aluminum plate. 
As illustrated in Fig. 2 (c), the magnetic flux lines generated by each turn are decomposed into roughly six components. Self-inductance is related to the sum of all six components. Mutual inductance is related to the sum of d, e and f. Component $\mathrm{f}$ is the source of LMF. Copper loss is mainly affected by components $\mathrm{a}, \mathrm{b}$ and $\mathrm{c}$. The coil assembly's geometric parameters influence all six flux components in complex ways.

At high frequencies, copper loss is increased due to skin effect and proximity effect. With the adoption of Litz wires, skin effect can be significantly diminished. By contrast, the proximity-effect-induced loss in each strand is caused by the magnetic field generated by all other strands, including those in the same turn and the rest that belong to other turns. Therefore, optimization of the coil pitch is required to reduce the coil ESR.

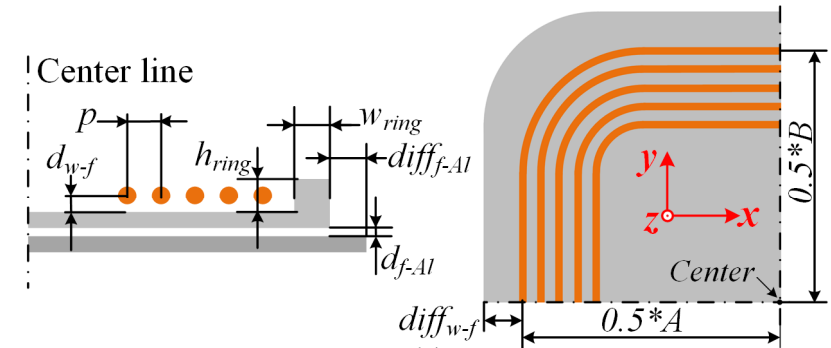

(a)

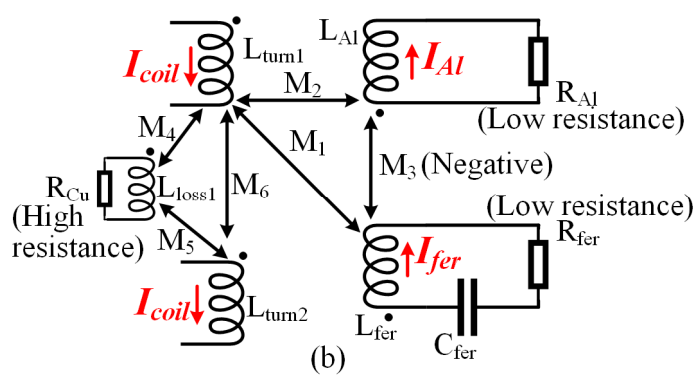

(b)

Fig. 3. Geometric parameters of the coil assembly and the electric-circuit analogy. (a) Geometric parameters. (b) Electriccircuit analogy of the magnetic circuit.

Fig. 3 (a) shows the main geometric parameters of the coil assembly. Based on the interactions among copper wire, ferrite core and aluminium plate, an electric-circuit analogy of the coil assembly as presented in Fig. 3 (b) is derived. $M_{1}, M_{2}, M_{4}, M_{5}$ and $M_{6}$ are positive mutual inductances whereas $M_{3}$ is negative. The equivalent inductance of the ferrite core $\left(L_{f e r}\right)$ is compensated by a series capacitance $\left(C_{f e r}\right)$ to such an extent that the loop impedance is capacitive. $R_{f e r}$ and $R_{A l}$ are small resistances utilized to characterize the core loss and eddy loss, respectively. Copper loss is modelled using an inductance-resistance loop with a high resistance $\left(R_{C u}\right)$. The total coil ESR is affected by the virtual mutual inductances and resistances. It is noteworthy that the $L_{l o s s 1}-R_{C u}$ loop is used to characterize the loss in $L_{t u r n 1}$ only: the proximity effect caused by $L_{t u r n 2}$ is modelled using $M_{5}$ and the other factors are modelled using $M_{4}$. The coil self-inductance, the actual mutual inductance in Fig. 1 and the LMF strength are mainly affected by $M_{1}, M_{2}, M_{3}$ and $M_{6}$. $I_{\text {coil }}$ is the actual coil current. $\boldsymbol{I}_{\boldsymbol{A} \boldsymbol{l}}$ lags $\boldsymbol{I}_{\boldsymbol{c o i l}}$ by roughly 180 degrees in phase, thus reducing both the actual mutual inductance and the self-inductance. By contrast, $\boldsymbol{I}_{\boldsymbol{f e r}}$ has almost the same phase as $\boldsymbol{I}_{\text {coil }}$ and improves both the actual mutual inductance and the self-inductance. The reason for the negative value of $M_{3}$ is that $\left|I_{\text {coil }}\right|$ is generally decreased when better magnetic shielding, i.e., a larger $\left|\boldsymbol{I}_{\text {fer }}\right|$, is available.

\subsection{Impact of Geometric Parameters on the FOMs}

FEA is conducted using the ANSYS Electronics Desktop 2020 R2 software. A useful feature of this software is that Litz wires can be directly modelled, which is especially helpful for copper loss estimation. The conductivity of copper and aluminium is set to $5.8 \times 10^{7}$ siemens $/ \mathrm{m}$ and $2.7 \times 10^{7}$ siemens $/ \mathrm{m}$, respectively. Based on practical parameters, the Litz wire diameter is set to $2.45 \mathrm{~mm}$ and the strand number is set to 600 . The relative permeability of ferrite (PC40) is set to 2200 and the core loss density is estimated using the following equation:

$$
P_{c v}=18.7176 f^{1.248} \hat{B}^{2.667} .
$$

The units of $P_{c v}$, fand $\widehat{B}$ are $\mathrm{W} / \mathrm{m}^{3}, \mathrm{~Hz}$ and Tesla, respectively. The coefficients are automatically calculated by the software after the loss curves (at 60 degrees Celsius) obtained from the datasheet (provided by TDK corporate) are imported. One can infer from (5) that $P_{c v}$ is roughly proportional to $\left|\boldsymbol{I}_{\text {coil }}\right|^{2.667}$. By contrast, 
copper loss and eddy loss are roughly proportional to $\left|\boldsymbol{I}_{\text {coil }}\right|^{2}$. Accordingly, the coil ESR is a monotonically increasing function of $\left|\boldsymbol{I}_{\text {coil }}\right|$. In the simulations, $\left|\boldsymbol{I}_{\text {coil }}\right|$ is fixed at $20 \mathrm{~A}$.

The geometric parameters shown in Fig. 3 (a) are varied to reveal their impacts on the FOMs. The majority of data are obtained at the zero-misalignment position unless when the impact of coil misalignment is emphasized, so that a quarter model instead of a full model can be used, which significantly reduces the burden on the computer. The mesh is refined until the changes in the simulation results brought by further refinement are insignificant.

The dimensions of the RX- and TX-side aluminium shielding plates are fixed at $600 \mathrm{~mm} \times 600 \mathrm{~mm} \times 2 \mathrm{~mm}$ and $300 \mathrm{~mm} \times 300 \mathrm{~mm} \times 5 \mathrm{~mm}$, respectively. The ferrite thickness is fixed at $5 \mathrm{~mm}$. The vertical distance between the TX coil and the RX coil is fixed at $130 \mathrm{~mm}$. For the sake of simplicity, the rounded corners in the copper winding are removed in the FEA model. The parameters of the original design are as follows: $p=3 \mathrm{~mm}, A=$ $270 \mathrm{~mm}, B=270 \mathrm{~mm}, d_{w-f}=3 \mathrm{~mm}, d_{f-A l}=2 \mathrm{~mm}$, diff $f_{w-f}=10 \mathrm{~mm}$, diff $f_{f-A l}=5 \mathrm{~mm}, h_{\text {ring }}=$ $0 \mathrm{~mm}, w_{\text {ring }}=5 \mathrm{~mm}$. Unless otherwise specified, only one parameter deviates from the initial value during the simulations.
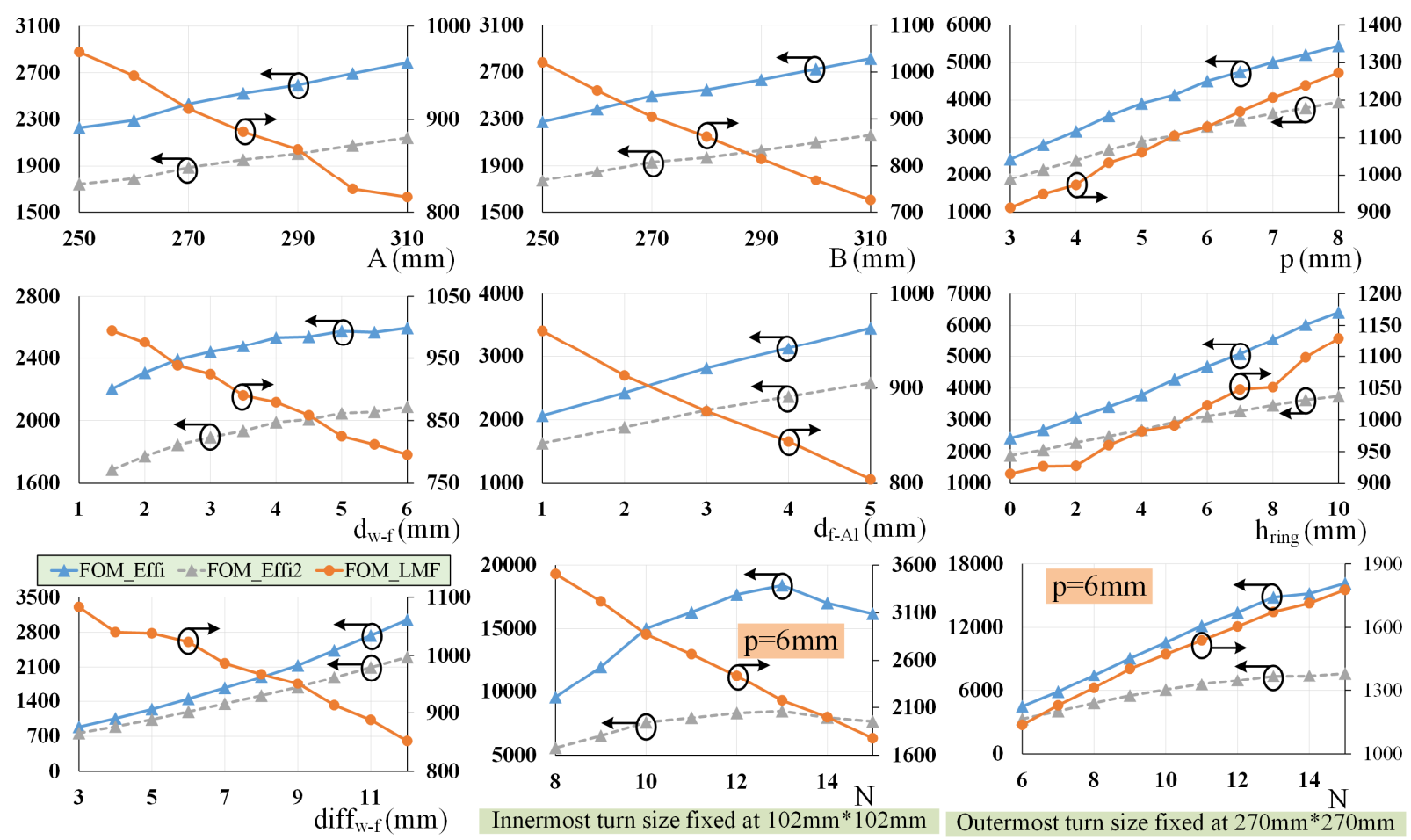

Fig. 4. Simulated FOMs.

Key observations obtained from the simulation results are listed below. (1) With the increase of inter-layer distances $\left(d_{w-f}\right.$ and $\left.d_{f-A l}\right), F O M_{L M F}$ is reduced while $F O M_{e f f i}$ is enhanced. (2) Increasing the coil assembly size (A or B) or the ferrite-coil size difference $\left(\operatorname{diff}_{w-f}\right)$ has the same effects as increasing $d_{w-f}$ does. (3) Adding a ferrite ring, i.e., protrusions along the outer contour of the ferrite layer, improves both FOMs. By contrast, the impact of $w_{\text {ring }}$ is small. (4) Increasing the coil pitch (p) is beneficial for improving both FOMs and the impact is significant. (5) When the outermost turn is fixed, increasing the turn number $(\mathrm{N})$ is beneficial for improving both FOMs. When the innermost turn is fixed, however, $\mathrm{N}$ exceeding a threshold reduces both FOMs. (6) When the total ferrite consumption is given, using an integral ferrite layer leads to higher FOMs than using multiple ferrite strips with obvious spaces in between. Part of the simulation results are presented in Fig. 4. Considering that core loss increases faster than copper loss when the excitation current 
is increased, core loss is artificially increased tenfold in deriving $F O M_{\text {effi2 }}$ to simulate higher-power operating conditions. When calculating the FOMs, the unit of $\mathrm{M}, R_{1 / 2}$ and $k_{1 / 2}$ is $\mu \mathrm{H}, \Omega$ and $\mu \mathrm{T} / \mathrm{A}$, respectively.

\subsection{LMF Suppression via Increasing the Aluminum Plate Size}

The largest coil misalignment is set to $70 \mathrm{~mm}$ in this work. To reduce the model complexity, coil misalignment is confined to the $y$-axis direction only, so that a half model instead of a full model can be used. Due to the coil misalignment, the LMF distribution is no longer symmetrical with respect to the $\mathrm{x}$-axis, hence the LMF strength is evaluated on two planes located $700 \mathrm{~mm}$ away from the RX coil centre and the lower FOM $L M F$ (i.e., the worst case) is considered. The normal vectors of the measurement planes are parallel with the y-axis. Simulation results show that $F O M_{L M F}$ can be easily improved by increasing the aluminium plate width (along the y-axis). Meanwhile, the negative impact on $F O M_{e f f i}$ is insignificant after the Al plate width exceeds $340 \mathrm{~mm}$, as is shown in Fig. 5.

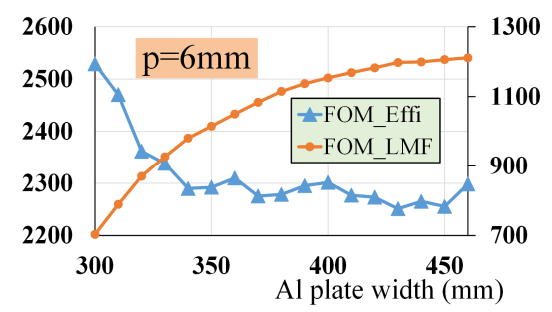

Fig. 5. Impact of Al plate width on the FOMs (coil misalignment is fixed at 70mm along the y-axis direction).

\section{Optimization of Coil Assemblies}

The objectives of coil assembly optimization are to increase both FOMs, particularly FOM effi (even at the expense of slightly lowering $F O M_{L M F}$ ), and enhance the coupling coefficient to reduce the reactive power. Due to the large number of geometric parameters and the complex relations among the FOMs and the geometric parameters, the optimal design depends heavily on the specific application. There are some parameters that have opposite impacts on both FOMs, e.g., A, B, diff $f_{w-f}, d_{w-f}$ and $d_{f-A l}$. Such parameters should be determined based on whether LMF suppression or efficiency improvement is of higher priority. Some parameters, e.g., p, N (when the outermost turn size is fixed) and $h_{\text {ring }}$, improve both FOMs when they are increased. Such parameters should be increased to a suitable level. The requirements on coil assemblies vary greatly from one system to another, hence the objective of this work is to analyse the possible means of improving the FOMs rather than obtaining an optimal design for a specific system.
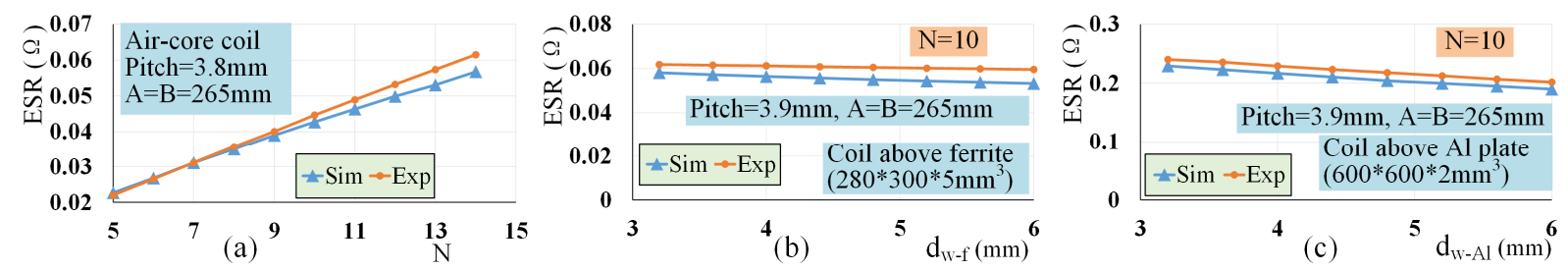

Fig. 6. Comparisons between simulated and measured coil ESR. (a) ESR versus N. (b) ESR versus $d_{w-f}$. (c) ESR versus $d_{w-A l}$ (distance between copper wire and aluminum plate).

Comparisons are made between FEA results and experimental results to verify the accuracy of the former, as is presented in Fig. 6. In the FEA model, the excitation currents in both coils have a constant amplitude of 20A. In the experimental measurements, the excitation current provided by the LCR meter (HIOKI IM3536) is 
$50 \mathrm{mArms}$ (at $85 \mathrm{kHz}$ ). Although the numbers do not exactly match, the overall trend obtained from FEA is credible.

During the optimization procedure, the vertical distance between the TX coil and the RX coil is fixed at $130 \mathrm{~mm}$, the TX- and RX-side Al plate dimensions are fixed at $300 \mathrm{~mm} \times 350 \mathrm{~mm} \times 5 \mathrm{~mm}$ and $600 \mathrm{~mm} \times 600 \mathrm{~mm} \times 2 \mathrm{~mm}$, respectively. A manually optimized design (quarter model) and the reference design are shown in Fig. 7. Except for the Al plate size, the TX and RX coil assemblies have identical parameters. For the sake of clarity, the RXside coil assembly is not shown.

With $70 \mathrm{~mm}$ coil misalignment along the y-axis, the mutual inductance in the reference and optimized designs drops by $28.5 \%$ and $22.3 \%$, respectively, which shows the stability of mutual inductance in the optimized design is better. In either the zero-misalignment case or the $70 \mathrm{~mm}$-misalignment case, both FOMs are higher in the optimized design.

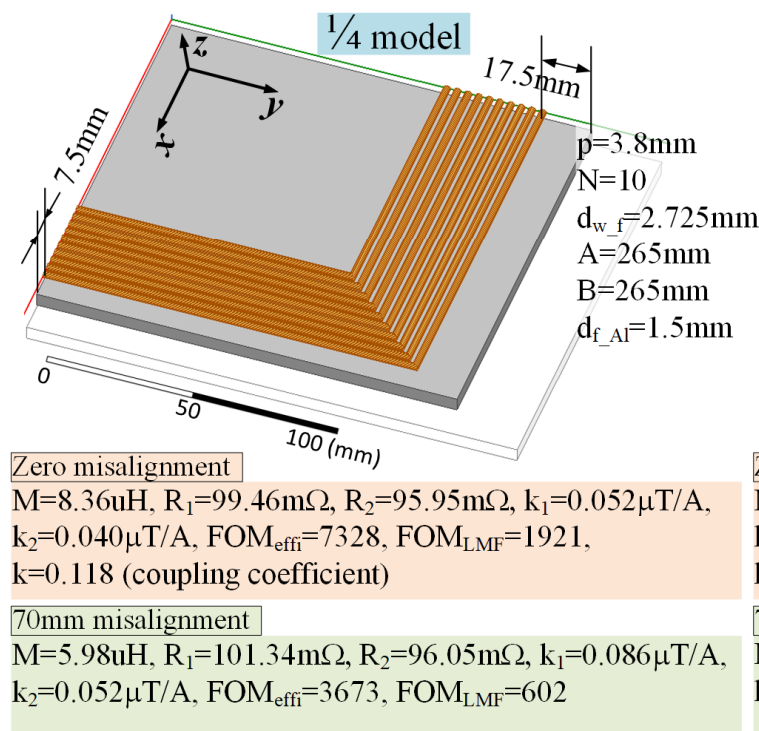

(a)

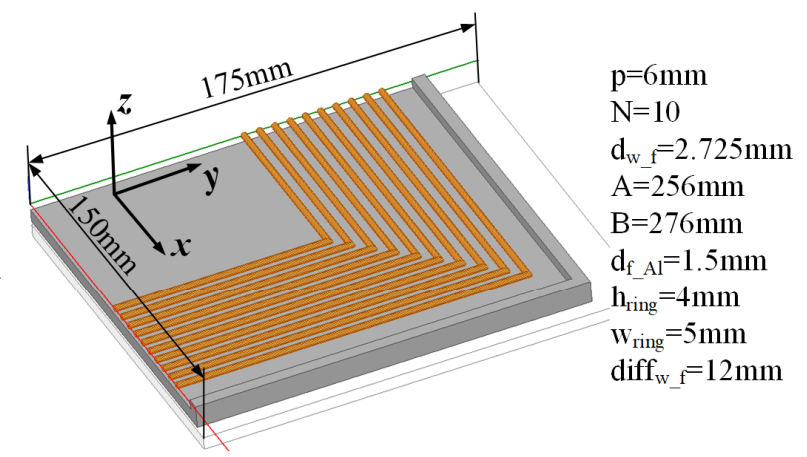

Zero misalignment

$\mathrm{M}=8.60 \mathrm{uH}, \mathrm{R}_{1}=65.68 \mathrm{~m} \Omega, \mathrm{R}_{2}=61.71 \mathrm{~m} \Omega, \mathrm{k}_{1}=0.053 \mu \mathrm{T} / \mathrm{A}$, $\mathrm{k}_{2}=0.038 \mu \mathrm{T} / \mathrm{A}, \mathrm{FOM}_{\mathrm{effi}}=18248, \mathrm{FOM}_{\mathrm{LMF}}=2057$, $\mathrm{k}=0.15$ (coupling coefficient)

$70 \mathrm{~mm}$ misalignment

$\mathrm{M}=6.37 \mathrm{uH}, \mathrm{R}_{1}=65.55 \mathrm{~m} \Omega, \mathrm{R}_{2}=61.58 \mathrm{~m} \Omega, \mathrm{k}_{1}=0.082 \mu \mathrm{T} / \mathrm{A}$,

$\mathrm{k}_{2}=0.054 \mu \mathrm{T} / \mathrm{A}, \mathrm{FOM}_{\mathrm{effi}}=10046, \mathrm{FOM}_{\mathrm{LMF}}=665$

Fig. 7. FEA models and simulation results (zero misalignment). (a) Reference design. (b) Manually optimized design.

\section{Conclusion}

Two FOMs are proposed for evaluating the performance of coil assemblies targeted at low-power electric vehicle inductive charging applications. The impacts of the main geometric parameters on both FOMs are revealed using finite element analysis, based on which a manual optimization procedure is conducted. In the optimized design, both FOMs are enhanced and the stability of mutual inductance against coil misalignment is improved, which is validated through FEA simulations. The following three measures can improve both FOMs: (1) maintain a relatively large coil pitch, (2) add a ferrite ring along the outer contour of the ferrite layer and (3) increase the turn number to a suitable value when permitted.

\section{Acknowledgments}

This work was supported by Beijing Natural Science Foundation (No. 3212030). 


\section{References}

[1] X. Dai, X. Li, Y. Li and A. P. Hu, Maximum Efficiency Tracking for Wireless Power Transfer Systems With Dynamic Coupling Coefficient Estimation, IEEE Transactions on Power Electronics, vol. 33, no. 6, pp. 50055015, June 2018

[2] G. Zhu, D. Gao and S. Lin, Leakage Magnetic Field Suppression Using Dual-Transmitter Topology in EV Wireless Charging, Journal of Power Electronics, vol. 19, no. 3, pp. 625-636, May 2019

[3] J. P. K. Sampath, A. Alphones and D. M. Vilathgamuwa, Coil optimization against misalignment for wireless power transfer, 2016 IEEE 2nd Annual Southern Power Electronics Conference (SPEC), Auckland, New Zealand, 2016, pp. 1-5

[4] R. Bosshard and J. W. Kolar, Multi-Objective Optimization of $50 \mathrm{~kW} / 85 \mathrm{kHz}$ IPT System for Public Transport, IEEE Journal of Emerging and Selected Topics in Power Electronics, vol. 4, no. 4, pp. 1370-1382, Dec. 2016

[5] Y. Yashima, H. Omori, T. Morizane, N. Kimura and M. Nakaoka, Leakage magnetic field reduction from Wireless Power Transfer system embedding new eddy current-based shielding method, 2015 International Conference on Electrical Drives and Power Electronics (EDPE), Tatranska Lomnica, Slovakia, 2015, pp. 241245

[6] M. Lu and K. D. T. Ngo, A Fast Method to Optimize Efficiency and Stray Magnetic Field for Inductive-PowerTransfer Coils Using Lumped-Loops Model, IEEE Transactions on Power Electronics, vol. 33, no. 4, pp. 3065 3075, April 2018

\section{Authors}

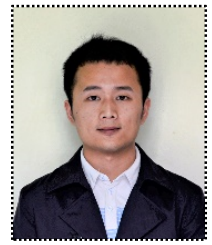

Guodong Zhu was born in Zhejiang, China, in 1995. He received the B.S. degree in Automotive Engineering from Tsinghua University, Beijing, China, in 2017, where he is currently working toward the Ph.D. degree in Power Engineering and Engineering Thermo-physics. His current research interest is wireless power transfer, particularly focusing on high-power electric vehicle wireless charging technology.

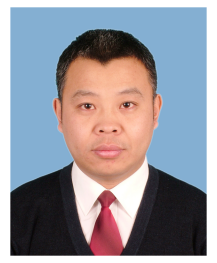

Dawei Gao was born in Jilin, China, in 1971. He received the B.S. degree from Southwest Jiaotong University, Sichuan, China, in 1992 and the Ph.D. degree from North China Electric Power University, Beijing, China, in 2001. From 2001 to 2003, he was a Postdoctoral Researcher with the Department of Automotive Engineering, Tsinghua University. From 2003 to 2005, he was an Assistant Researcher. Since 2005, he has been an Associate Researcher with the same department. His research interests include DC-DC converters, electric vehicle wireless power transfer, automotive power electronics and electric drive, and electric vehicle powertrain optimization. 